Proceedings of the 2011 Winter Simulation Conference

S. Jain, R.R. Creasey, J. Himmelspach, K.P. White, and M. Fu, eds.

\title{
CHALLENGES FOR WEB SIMULATION SCIENCE
}

\author{
Simon J. E. Taylor \\ ICT Innovation Group \\ School of Information Systems, Computing and Mathematics \\ Brunel University, Uxbridge, UB8 3PH, UK
}

\begin{abstract}
Web Simulation Science may be viewed as part of the emerging discipline of Web Science. Essentially combining the Semantic Web and Hypermodeling with Modeling \& Simulation, this area presents the opportunity for M\&S to fully exploit the advantages of model ontologies, discovery, composition, interoperability and reuse in a revolutionary way. However, was this not also the promise of Web-based Simulation? This paper discusses what benefit Web Simulation Science could bring to M\&S and what challenges this area must overcome to make a significant impact.
\end{abstract}

\section{INTRODUCTION}

The Semantic Web is revolutionizing the World Wide Web and has brought with it issues such as ontology engineering, ontology alignment, ontology mapping, discovery, composition, and interoperability. The Web can be considered as a complex ecosystem of artifacts. Web Science studies this and its emergent complexity (Hendler et al. 2008). In Modeling and Simulation (M\&S) the Semantic Web may have a significant effect on methods and tools, particularly in model ontologies, discovery, composition, interoperability and reuse. Hypermodeling, the general theory and practice of linking system models and their components, has been proposed by Fishwick (2011). Combining this with Web Science arguably gives us the embryonic field of Web Simulation Science (WSS). Can WSS make an impact?

There is a feeling of déjà vu. Web-based Simulation emerged in the mid-1990s. This was put forward as an exciting new area that could fundamentally change the practice of M\&S. Until recently, the technologies used in $M \& S$ have remained stubbornly non-Web-based. Now most major M\&S tools, or COTS Simulation Packages, such as AnyLogic and Simul8, have web-based aspects. However, these have aspects more commonly associated with Groupware (Taylor 2000) and certainly do not seek to take advantage of model/component discovery, composition, interoperability and reuse (Taylor et al. 2010).

As evidenced by the special issue on simulation and ontologies (a major component of WSS) (Tolk and Miller 2012), there is a significant amount of activity in the area. This was also the case with Webbased Simulation. Are the technological aspects of Web Simulation Science another 'flash in the pan'? Anyone who has made even a quick cursory will see that the Semantic Web is not a simple 'rebranding' of the World Wide Web. It brings with it new ways of thinking about modeling and new methods and tools. The largest immediate impact may well be in new socio-technical studies of 'template' models or reusability that have been proposed, i.e. a modeler 'grabs' different model objects from different sources and 'glues' them together to make a new model (Paul and Taylor 2002). Are Emergency Room models all that different? Are most manufacturing systems basically the same? Is the same true for most business processes?

Web-based Simulation faced many barriers to its use. In some ways Distributed Simulation is facing similar issues. Arguing that these are examples of (in their time) new, complex technologies that are (were) being applied to industry, this paper draws upon my experiences of these technologies and presents a set of challenges that may need to be considered when Web Simulation Science is used to study and guide the realization of new approaches to $M \& S$. 


\section{Taylor}

\section{WEB SIMULATION SCIENCE}

Web Science studies the vast socio-technological issues of the Web as an entity in its own right (Hendler et al. 2008). As presented by the Web Science Trust,

"The Web is the largest human information construct in history. The Web is transforming society. In order to understand what the Web is, engineer its future and ensure its social benefit we need a new interdisciplinary field that we call web science."

There are strong parallels with M\&S, albeit on a smaller scale. For example, in a M\&S project there are many different elements that are brought together to investigate a particular problem or perform a given task (the informational artifacts brought together to simulate a production line, a hospital or military scenarios). As outlined above, attempts have been made to use the Web to transform M\&S. Arguably $\mathrm{M} \& S$ is being applied to larger and larger problems. So in order to "engineer its future" and "ensure its benefit" I argue we need a new field that is called Web Simulation Science (WSS).

I define WSS as the study of M\&S ecosystems realized through Semantic Web and Hypermodeling technologies. An M\&S ecosystem is 'everything'; the emergent complexity of people, organizations and society with regard to $\mathrm{M} \& \mathrm{~S}$ artifacts (models, tools, technology, data, etc.). A key component to this is Hypermodeling which subsumes and extends approaches to connect and integrate simulation models (Fishwick 2011). It is strongly associated with the notion of hyperdocument, a media form that interrelates information and media to extend previously static interpretations of documents. Hypermodeling covers:

- Linking models together within one computer or among many

- Linking system models to other types of models (e.g., information, geometry)

- Linking model components to media

- Composing system models from heterogeneous model types

- Creating custom representations of the same formal model

For contributions to M\&S with respect to each of these the reader is referred to Fishwick's articles as cited above. The potential applications of Hypermodeling are vast. WSS could not only study and guide novel M\&S technology but also inform us about the complex interplay between modeler, stakeholders and $M \& S$ artifacts (tools, models, data, etc.) in contemporary M\&S practice. Equally, there are many challenges that could result in WSS not making any significant contribution at all.

\section{CHALLENGES FOR WEB SIMULATION SCIENCE}

The following lists a set of key challenges for WSS based on personal experiences of new technological development over a 20 year period (see, for example, Miller et al. 2001; Mustafee and Taylor 2009; Robinson et al. 2004; Taylor and Robinson 2006; Taylor et al. 2006; Taylor et al. 2010). These include experiences in Web-based Simulation, Distributed Simulation and Grid Computing. These are presented below and have, unsurprisingly, some overlap. I leave the reader to order these as they wish.

- Ease of use. Some aspects of Hypermodeling are extremely complex and still only implementable with considerable effort e.g. (time management in distributed simulation, ontological languages). Realistically, is it possible to predict whether or not these complex aspects can ever be solved? This might help to galvanize and focus research.

- Clear use/business case. The motivation for using new technologies in M\&S is sometimes based only on the needs of the researcher (research for research's sake). Clearly articulated real world use and/or business cases need to be defined to give (a) clear research 'target and (b) clearly show the relevance of the research. If this cannot be done then one may question if the research is worth pursuing. 


\section{Taylor}

- Relevant demonstrators. Following on from above, use cases must be articulated by technology demonstrators that are clearly relevant to end users. Even if these are 'mock ups' it is vital so that end users can see complex ideas in a relevant setting.

- Language/world view. M\&S practitioners speak a different 'language' or have a different world view to many researchers. Researchers must take care to properly understand the motivations and needs of M\&S end users for any new approach/technology to be realized. Such new artifacts must be capable of being expressed in terms that $M \& S$ practitioners find accessible.

- Appropriate problem complexity. Overly simple applications are useful in developing demonstrators. However, solutions for this must be capable of being scaled to up levels of appropriate problem complexity to show 'worth'.

- Understanding of contemporary M\&S technology. Many M\&S practitioners use COTS simulation packages. It is highly unlikely that any new approaches will ever be taken seriously unless they can be 'expressed' in terms of COTS simulation packages, etc.

- Understanding of stakeholders. In M\&S there are many stakeholders, beneficiaries of M\&S project outcomes, modelers, data owners, system stakeholders, software vendors. In the development of new technologies all these stakeholders may have a role to play (some major, some minor). Not properly understanding these may have a negative effect on technological development.

- Understanding the problem domain. Following the above it can be problematic for researchers to assume that one $M \& S$ community is the same as another. For example, certain technologies have been developed for different M\&S communities and may not be 'translatable' (e.g., military to manufacturing to health).

- Adopting a multidisciplinary approach. There is almost no published work on M\&S in social science, i.e. socio-technical studies of M\&S as a social phenomenon. M\&S research teams tend to be operational researchers, management scientists, computer scientists, engineers or domain experts/SMEs. Social scientists have deep insight into the complexities of human interaction. A lack of understanding of this, particularly from an adoption of new technologies viewpoint, might be critical.

- Standards. Standards exist in many different areas and associated areas of M\&S. In M\&S, the majority is in distributed simulation and distributed simulation related areas. The Web has a plethora of standards (SOA/Web Service standards for example). Domain data standards can be huge as can CAD standards. There are several aspects to this. Web technologies are developed in a framework of standards and care must be taken to ensure that these technologies use the 'right' ones (although there is some debate on this). Some standards change, some are 'deleted'. Solutions must reflect this. Ultimately, any web-based technology will be a 'slave' to standards or be a leader to/creator of a standard. New technologies must clearly link to standards otherwise development may be flawed or naïve.

- Organizational settings. New M\&S technologies will be used in different industrial sectors. These have managed IT environments which are sometimes more restrictive that research IT environments. While one cannot predict every possible situation, solutions should be developed will some 'organizational' use case in mind. For example, in some settings models must be treated as black boxes, the internal workings may be confidential. Further the model may not be able to leave the boundary of the organization. Similar observations can be made on data.

- M\&S Methodology. New technologies can be developed with new methodologies in mind. However, one must recognize that, unless there really is a Web revolution, it is highly likely that future M\&S practices will be similar to those used now. New approaches must blend in to contemporary ones to build credibility. If this can be achieved then perhaps a quiet revolution could occur.

- Re-inventing the wheel. Researchers creating new technological artifacts sometimes do this without understanding the state-of-the-art. In the past this has led to a series of unrelated isolated 


\section{Taylor}

developments that all attempt to solve the same problem. While innovation itself is to be encouraged, poor research methodology is not!

- Research rigor. Successful ground breaking technology is often created on a firm foundation of theory and practice. This firm foundation comes usually in the form of peer-reviewed conference and journal papers in quality conferences. In the absence of broad base commercialization, this foundation is critical for well-informed debate and development. Note that a problem in M\&S publishing is low number of papers that cite end user involvement (in the development and/or implementation of a solution) (Taylor et al. 2009). How can one demonstrate the positive impact of new technology without some end user evaluation?

- Critical mass/sustainability. Research often requires funding. When we receive funding we focus on a problem and move the solution to that problem forward. When funding is running out we attempt to get more funding to carry on the research. Invariably if that funding does not come, the research stops. In a new (any!) area it is very difficult to pursue long term sustainable research. It is often very difficult to make a long term commitment to advancing a specific area in a specific field. One may hope that a critical mass of researchers will come together to share and sustain research efforts. This is possible but only with careful (and competitive) planning.

- Dissemination. Careful and targeted communication of the benefits of new technologies has to be communicated to target end user communities. One cannot assume that by creating a new technology/approach end users will come 'flocking'. Instead one must carefully plan a dissemination strategy to communicate the benefits directly to the end user community. Better if one can convince a key member of that community to communicate benefits directly.

- Appreciate the end users of tomorrow. School leavers entering higher education have different IT experiences to perhaps current M\&S users. Although this has been acknowledged, has this ever been seriously studied? This might realistically portray the expectations of tomorrow's M\&S community rather than one presented by an older generation.

\section{CONCLUSIONS}

This brief article has outlined and defined Web Simulation Science and Hypermodeling. It has drawn upon my experiences to present a set of challenges that may need to be considered when Web Simulation Science is used to study and guide the realization of new approaches to M\&S. Both Web Simulation Science and Hypermodeling may well have a significant role to play in M\&S. I encourage researchers to look into the past and revisit Web-based simulation literature. There are relevant ideas and concepts that could well be applied to contemporary research efforts. I also encourage researchers to attempt to bring scientific rigor to Hypermodeling and through the development of Web Simulation Science so that it can guide $\mathrm{M} \& \mathrm{~S}$ efforts in the future.

\section{REFERENCES}

Fishwick, P.A. 2011. "Hypermodeling: An Integrated Approach to Dynamic System Modeling." Journal of Simulation. In press.

Hendler, J., N. Shadbolt, W. Hall, T. Berners-Lee and D. Weitzner. 2008. "Web Science: An Interdisciplinary Approach to Understanding the Web." Communications of the ACM, 51(7) 60-69.

Paul, R. and S. Taylor. 2002. "What Use is Model Reuse: Is There a Crook at the End of the Rainbow?" In Proceedings of the 2002 Winter Simulation Conference, edited by E. Yücesan, C. H. Chen, J. L. Snowdon, and J. M. Charnes, 648-652. Piscataway, New Jersey: Institute of Electrical and Electronics Engineers, Inc.

Taylor, S.J.E. 2000. "Groupware and the Simulation Consultant." In Proceedings of the 2000 Winter Simulation Conference, edited by J. A. Joines, R. R. Barton, K. Kang, and P. A. Fishwick, 83-89. Piscataway, New Jersey: Institute of Electrical and Electronics Engineers, Inc. 
Taylor, S. J. E., D. Bell, N. Mustafee, S. de Cesare, and P.A. Fishwick. 2010. "Semantic Web Services for Simulation Component Reuse and Interoperability: An Ontology Approach." In Organizational Advancements through Enterprise Information Systems: Emerging Applications and Developments, edited by A. Gunasekaran and T. Shea. IGI Global, Inc., Chapter 21, 336-352.

Taylor, S.J.E., T. Eldabi, G. Riley, R.J. Paul and M. Pidd. 2009. "Simulation modelling is 50! Do we need a reality check?" Journal of the Operational Research Society, 60(1) S69-82.

Taylor, S.J.E. and S. Robinson (2006) "So where to next? A Survey of the Future for Discrete-Event Simulation." Journal of Simulation. 1(1) 1-6.

Tolk, A. and J. Miller, editors. 2012. Journal Of Simulation, Special Issue: Enhancing Simulation Composability and Interoperability using Conceptual/Semantic/Ontological Models, 5(3).

Robinson, S., R. Nance, R.J. Paul, M. Pidd and S.J.E. Taylor. 2004. "Simulation Model Reuse: Definitions, Benefits and Obstacles." Simulation Modelling Practice and Theory, 12, 479-494.

Miller J., P.A. Fishwick, S.J.E. Taylor, B. Benjamin and B. Szymanski. 2001. "Research and Commercial Opportunities in Web-Based Simulation." Simulation: Practice and Theory, 9(1-2), 55-72.

Mustafee, N., and S. J. E. Taylor. 2009. "Speeding Up Simulation Applications Using WinGrid." Concurrency and Computation: Practice and Experience, 21(11), 1504-1523.

Taylor, S.J.E., X. Wang, S.J. Turner, and M.Y.H Low. 2006. "Integrating Heterogeneous Distributed COTS Discrete-Event Simulation Packages: An Emerging Standards-Based Approach." IEEE Transactions on Systems, Man \& Cybernetics: Part A. 36(1), 109-122.

Taylor, S.J.E, N. Mustafee, S. Kite, C. Wood, S. J. Turner, and S. Strassburger. 2010. "Improving Simulation through Advanced Computing Techniques: Grid Computing and Simulation Interoperability." In Proceedings of the 2010 Winter Simulation Conference, edited by B. Johansson, S. Jain, J. Montoya-Torres, J. Hugan, and E. Yücesan, 216-230. Piscataway, New Jersey: Institute of Electrical and Electronics Engineers, Inc.

Web Science Trust.www.webscience.org.

\section{AUTHOR BIOGRAPHIES}

SIMON J. E. TAYLOR is the Founder and Chair of the CSPI PDG under SISO. He is the co-founding Editor-in-Chief of the UK Operational Research Society's (ORS) Journal of Simulation and the Simulation Workshop series. He was Chair of ACM's SIGSIM (2005-2008). He is a Reader in the School of Information Systems, Computing and Mathematics at Brunel and leads the ICT Innovation Group. He has published over 100 articles in modelling and simulation. His recent work has focused on the development of standards for distributed simulation in industry. His email address is <simon.taylor@brunel.ac.uk>. 